\title{
Mucocoele Of The Appendix
}

\author{
K SAGGAR, AAHLUWALIA, P SANDHU, V KALIA
}

Ind J Radiol Imag 2006 16:2:191-192

Key words : Mucocele, Appendix, USG, CT

$\mathrm{M}$ ucocoeles of appendix are rare, appearing in 0.2 $-0.3 \%$ of surgical appendectomy specimens. They usually present as right lower abdominal pain. The male:female preponderance is $1: 4$. There may be complications like malignant change, pseudomyxoma peritonei and intusseption of mucocoele.

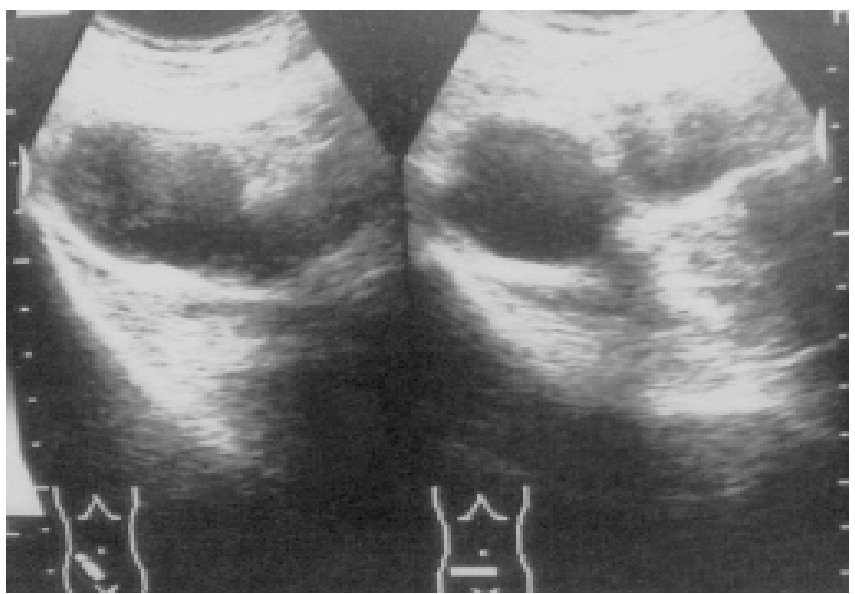

Fig. 1: High resolution ultrasound of right lower quadrant of the abdomen showing a cystic anechoic mass with posterior acoustic enhancement

A 65-year-old male presented with pain in the right lower quadrant. Physical examination was normal. Haemogram revealed no significant abnormality. Ultrasound Abdomen revealed a tubular anechoic cystic lesion in right lliac fossa with good through transmission measuring $6.0 \mathrm{x}$ $2.5 \mathrm{~cm}$. Contrast enhanced spiral CT of the abdomen showed well marginated thin walled tubular structure of fluid attenuation (13HU) posterior to the caecum. No calcification was seen in it. No inflammation of the surrounding fat was seen. There was no evidence of ascites. Provisional diagnosis of Mucocoele of appendix was made and was confirmed on surgery and histopathology.
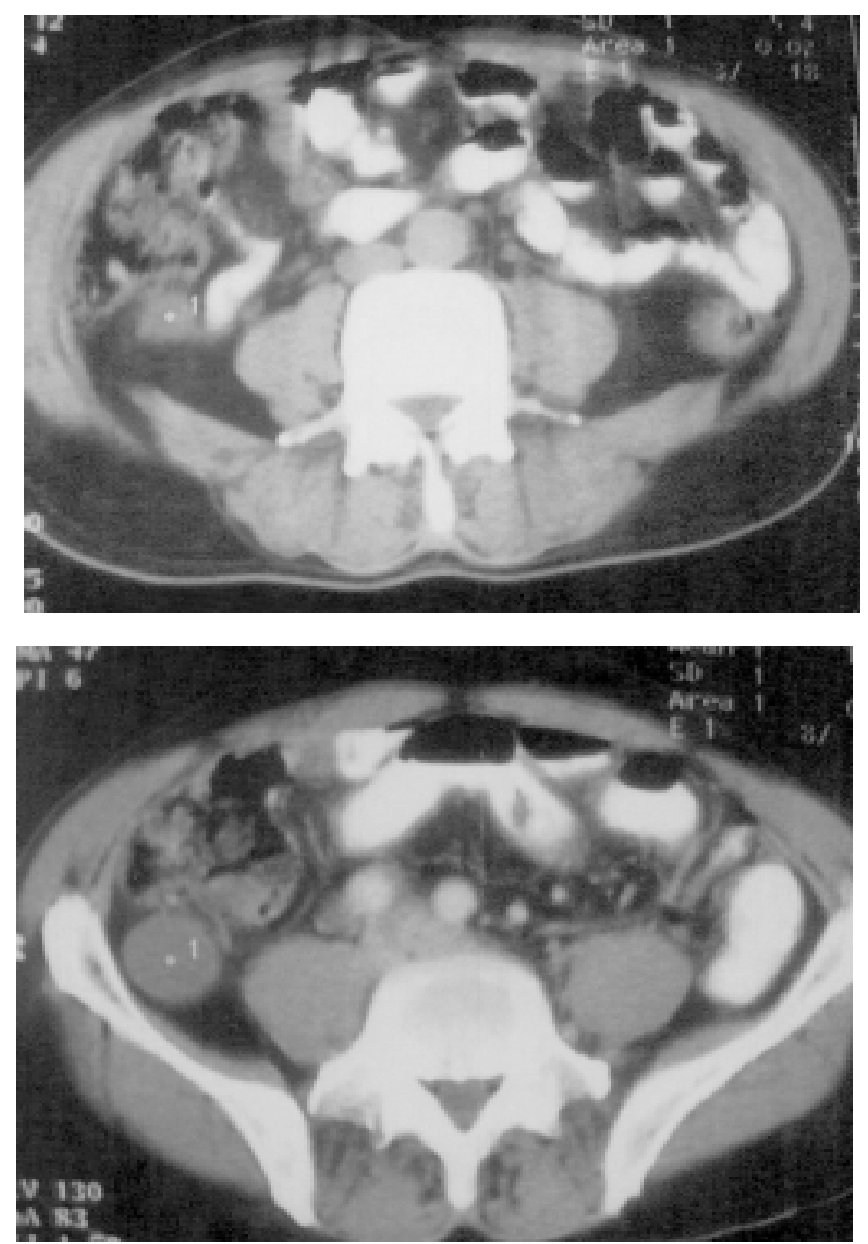

Fig. 2 \& 3:

CT sections showing low attenuating smooth lobulated mass in relation to the caecum suggestive of mucocoele of appendix.

The term mucocele of appendix was first described by Rokitansky in 1842 [2]. Higa et al preferred to consider all the mucocoeles as mucinous neoplasms within a clinico-pathological spectrum comprising of mucosal hyperplasia, mucinous cystadenoma and mucinous

From the Department of Radiodiagnosis, Dyanand Medical College \& Hospital, Ludhiana - 141001

Request for Reprints: Dr. Vishal Kalia, 464-sec 8/li, Urban Estate, Karnal, Haryana

Received 12 November 2005; Accepted 15 April 2006 
cystadenocarcinoma [3]. The term mucocoele has been retained by popular usage [4]. Euphrat defined its radiological criteria as follows:

a) Sharply defined circumscribed globular or reniform soft tissue mass, with considerable motility but firmly attached to the caecum

b) Medial displacement of the caecum by this mass [3]

c) Calcium deposits in the wall or substance of a mass.

d) Failure of the appendix to fill with contrast and vortical (concentric ring) appearance of the folds of caecal mucosa [5].

Plain radiographs of the abdomen may demonstrated a soft tissue mass in the right lower quadrant with or without calcification and usually appear curvilinear on plain film but may appear globular when viewed enface $[4,6]$.

On ultrasound, there is a cystic mass extrinsic to solid abdominal viscera. Polypoidal excrescence may be seen projecting intraluminally from the wall of mucocoele, possibly representing proliferation of appendiceal mucosa. There is typically excellent good through transmission and posterior wall enhancement. When wall is calcified, posterior acoustic shadowing may occur. The wall thickness varies but if it is more than $6 \mathrm{~mm}$, one should also consider uncomplicated acute appendicitis. Internal septations, polyploid lesions extending into the lumen and irregular shape seem to be associated with malignant variety, although some papillary processes may be seen in mucinous cystadenomas $[1,7,8]$

CT may show a low attenuation (0-40 HU) smooth or lobulated mass with or without calcification or septation. They may have simple or multiple cystic components and some solid element and may even infiltrate surrounding organs such as colon and bladder [1,9].

Rupture of the mucocoele may cause pseudomyxoma peritonei in which CT may show low attenuation ascites (5-20 HU; 1:1000 scale), scalloping of the liver margin and bowel loops due to extrinsic pressure by adjacent peritoneal implants. There may be intusseption of the appendix. In the presence of intusseption, a mucocoele may present as a soft tissue mass or as a calcification in the unusual location, which may mimic a gallstone or an enterolith. A barium enema may show the usual feature of intusseption or intraluminal mass which may mimic a carcinoma in the right colon $[3,4]$.

Myxoglobulosis is a variant of mucocoele seen in $0.35 \%$ to $8 \%$ of cases in which the appendix is filled with many solid translucent globules. It was first described by Latham [3]. When it calcifies, spherules $1-10 \mathrm{~mm}$ in diameter, which are generally uniform in size, may be seen on plain films and may shift with mucocoele. This appearance is said to be pathognomic when the calcification can be localized to the appendix [10].

The finding of appendiceal mucocoele should prompt a search for an association of adenocarcinoma of colon (6 times increase incidence). There are associations of mococele of the appendix with mucous secreting tumor of the ovary [1].

The differential diagnosis includes intraperitoneal or extraperitoneal lesions. Intraperitoneal masses to consider include ovarian cysts and tumours, duplication cysts, mesentric and omental cysts, mesentric hematoma or tumour, and abdominal abscess. Of the retroperitoneal disorders to be considered, retroperitonal inflammation, tumour or haemorrahages are important. Renal cyst and pancreatic pseudocyst also should be included.

Imaging usually differentiate between these entities and presence of a retrocaecal mass requires consideration of appendiceal pathology, as $65 \%$ of appendices lie in this position [1,4].

\section{References}

1) P. Yadav, S. Mukherjee, J.S.Slkarwar, G L Sharma. High resolution ultrasonographic diagnosis of mucocoele of Appendix. IJRI 2003; 13; 1:33-34.

2) Rokitansky CF. A manual of Pathological Anatomy. Vol.2 English Translation of Vienna edition (1842) Philadelphia. Blanchard \& Lea, 1855: 89.

3) Higa E, Radai J, Pizzimbono C A, Wise L. Mucosal Hyperplasia, Mucinous cystadenoma and mucinous cystadenocarcinoma of the appendix. Cancer 1973; 32: 1525-1541.

4) Abraham H. Dachman, Jod LE, Arnold CF. Mucocoele of Appendix and Pseudomyxoma Peritonei. AJR, May 1985; 923-929.

5) Euphrat EJ. Roentgen Features of Mucocoele of Appendix. Radiology 1947; 48:113-117.

6) J. Gerard Horgen, Peter PC, Joan OR, Arthur TR, Kenneth JW Taylor. Computed Tomography and Sonography in the recognition of Mucocoele of Appendix. AJR, Nov 1984; 143: 959-962.

7) Mad Wed D, Mindelzun R, and Jeffery RB Jr. Mucocoele of Appendix. Imaging Findings. AJR, July 1992; 159: 6972.

8) Dega ni S, Shapiro I, Leibovitz Z et al. Sonographic appearance of Appendiceal Mucocoele. J Ultrasound Obstet Gynecol 2002, 19 (1): 99-101.

9) Balthazar, Email J. CT of Abnormal Appendix. J Comput Assist Tomogr; July/ Aug, 1988; 12(4): 595-601.

10) Novitsky GJ, Berlin L, Epstein AJ, Lobo N, Mille SH. Case report: Pseudomyrene peritonei. J Comput Assist Tomogr 1982; 6: 398-399.

11) Latham. Cited in Prostein JG, Lasskr GN. Mucocoele of Appendix with Myxoglobulosis. Ann Surg 1948; 127: 171176. 